\title{
Stochastic and Deterministic Models in Epidemiology
}

\author{
Carlos Castillo-Chavez, $\uparrow$ \\ Samuel Fridman, $\ddagger$ and Xiaolong Luo*
}

\begin{abstract}
This article explores the relationship between stochastic and deterministic models in demography and epidemiology. We conduct a large number of simulations of our stochastic demographic pair-formation model and concentrate on the average behavior of the model and its variability. We provide an outline of future work in this area.
\end{abstract}

1991 Mathematics Subject Classification: 92D30.

\section{Introduction}

Classical demography $[21,22,25]$ ignores social dynamics and usually concentrates on the birth and death processes of female populations but ignores specific mating/contact structures. The incorporation of mating structures or marriage functions, as they are commonly referred to in human demography, was pioneered by both Kendall and Keyfitz $[17,19]$. Their work was extended by Parlett, Fredrickson, and others $[10,26,27,28]$ two decades ago. However, their impact on demography, epidemiology, and population biology has been minimal.

The grim scenario due to the HIV/AIDS epidemic has accelerated the pace at which social dynamics have been incorporated into epidemiological models. There has been intensive research activity on looking at the effects of social dynamics, immune system variability, etc. in HIV dynamics as well as on the development of methods for the evaluation of competing control measures.

The work that we (this generic "we" includes many collaborators, some of whom will be cited throughout the text) have conducted over the last few years has had as a major component the development of a mathematical framework for the systematic incorporation of very general contact structures. The modeling approach

$\dagger$ Biometrics Unit and Center for Applied Mathematics, 337 Warren Hall, Cornell University, Ithaca, NY 14853-7801

$\ddagger \quad$ Biometrics Unit, 337 Warren Hall, Cornell University, Ithaca, NY 14853-7801

* Department of Biostatistics, St. Jude Children's Research Hospital, 332 N. Lauderdale, P. O. Box 318, Mephis, TN 38101-0318 
outlined in this volume in the paper of Lubkin and Castillo-Chavez and in the one by Velasco-Hernández and Castillo-Chavez is being used to develop models for the study of disease dynamics, the dynamics of frequency-dependent predation in heterogeneously mixing populations (food web dynamics), transmission dynamics of cultural traits, social dynamics, general demographic processes, etc. In addition, we have made serious efforts to connect these models to data. Our data oriented work is being reported in the contribution by Hsu Schmitz and Castillo-Chavez.

This manuscript is organized as follows: Section 2 discusses the role of contact/social structures in demography and epidemiology, reviews some of the literature, and draws connections to other manuscripts in this series. Section 3 illustrates the connections between deterministic and stochastic processes. Section 4 collects the results of the simulations of a demographic two-sex stochastic model that follows the dynamics of pairs.

\section{On contact/social structures}

The contact or social structure of a population may have a fundamental role in the transmission dynamics of diseases, cultural traits, genetic traits, etc. It has been usually modeled by assuming that the rate of transmission of the trait in consideration is directly proportional to those that have it and to those that do not. This assumption is embodied in the mass-action law.

The mass-action law has played a central role in the development of stochastic and deterministic epidemiological models (see $[1,2,3]$, and references therein). The assumption that the rate of new infections (the incidence) is proportional to the product of susceptibles and infectives in the exposed population is not very limiting when dealing with interacting subpopulations of constant size except, perhaps, for the appropriate interpretation of relevant epidemiological parameters. However, the mass-action assumption is very limiting when dealing with interacting subpopulations of varying size (that is, when the sizes of the interacting subpopulations vary according to deterministic or stochastic rules). A thorough analysis of assumptions such as those implict in the mass-action law in epidemiology has been carried out in a systematic fashion (see [5,6]). From this work, one concludes that modelling the interactions between susceptible and infective individuals using the mass action law is not appropriate for many diseases.

The HIV/AIDS epidemic revealed the deficiencies and inadequacies of the existing theory-based on the mass-action law and proportionate mixing. Several questions relevant to the dynamics of heterogeneously mixing populations affected by fatal diseases could not be properly studied under the existing framework. The contact/social structure of the population must respond at least to potential demographic/epidemiological changes in the population due to a heterogeneously transmitted fatal disease (or, more generally, to frequency dependent predation). The importance of the contact process (well recognized by Ross in his work on malaria [29]) on frequency-dependent systems has motivated the work discussed 
by Velasco-Hernández and Castillo-Chavez in this series. The article by Lubkin and Castillo-Chavez, also in this series, addresses its use in a demographic framework.

The work of Lubkin and Castillo-Chavez on pair formation models provides an alternative to the early approach $[10,17,18,26,27,28]$ and its later extensions to epidemiology $[8,9,11,12,13,30]$. In the next section we introduce a stochastic analog to the model presented by Lubkin and Castillo-Chavez. The properties of such stochastic model are also explored in the next section. The approach of Dietz and Hadeler $[8,9,11,12]$ is based on the use of a nonlinear function $\psi$ to model the process (rate) of pair formation. This mixing/pair-formation function is assumed to satisfy the Fredrickson/McFarland $[10,26]$ properties, which are discussed in more detail in the contribution by Lubkin and Castillo-Chavez.

In the next section, we also discuss extensions of the mixing/pair-formation approach of Castillo-Chavez and Busenberg to models for sexually-transmitted diseases that follow pairs. Classical deterministic models for the sexual spread of STD's such as gonorrhea among heterosexual populations can be found in Hethcote and Yorke [14] while classical and pair formation models under a unified mixing framework for the spread of STD's can be found in Blythe et al. [4] and references therein. A stochastic version of the deterministic models found in [4] is provided below. Because this formulation uses the modeling approach common to interacting particle systems (for details, see $[23,24]$ ), it has great generality and flexibility.

\section{Stochastic pair formation models}

In this section, we introduce a stochastic pair formation model. The model formulation uses the approach that is common to interacting particle systems and, consequently, it offers great flexibility. From its description, extensions to model more complex scenarios should be quite evident. Here is the description of the model:

General Notation.

Let $X=\{0,1, \ldots, L\} \times\{0,1\} \times\{0,1, \ldots, N\} \times\{0,1\} \backslash\{0\} \times\{0,1\} \times\{0\} \times\{0,1\}$, and consider the explicit stochastic process $\xi_{t}: X \rightarrow\{0,1,2, \ldots\}, t \geq 0$. Then, for $x=(i, \mu ; j, \nu) \in X$, our interpretation of this process is as follows:

1. The labels $\mu$ and $\nu$ represent the epidemiological status of individuals. Specifically, $0 \equiv$ susceptible and $1 \equiv$ infected. The labels $i$ and $j$ represent groups of males and females;

2. For $i>0$ and $j>0, \xi_{t}(i, \mu ; j, \nu)$ gives the number of pairs where the male is of the $i$ th type and has epidemiological status $\mu$ and the female is of the $j$ th type and has status $\nu$ at time $t$;

3. Singles are labeled by triplets. However, to keep the domain fixed we use four coordinates and set either $i$ or $j$ equal to zero. Specifically, if $i>0$ and $j>0$, then $\xi_{t}(i, \mu ; 0) \equiv \xi_{t}(i, \mu ; 0,0) \equiv \xi_{t}(i, \mu ; 0,1)$ denotes the number of single males with status $\mu$ in the $i$ th subpopulation at time $t$. Similarly, if $i=0$ and $j>0$, 
then $\xi_{t}(0 ; j, \nu) \equiv \xi_{t}(0,0 ; j, \nu) \equiv \xi_{t}(0,1 ; j, \nu)$ denotes the number of single females with status $\nu$ in the $j$ th subpopulation at time $t$.

Let $S \equiv\{0,1,2, \ldots\}^{X}$ and let $c: S \times S \rightarrow(0, \infty)$ be a real-valued function, the flip rate, to be specified later. We view $\left\{\xi_{t}: t \geq 0\right\}$ as an $S$-valued Markov process with flip rate $c(.,$.$) , i.e., if \xi_{t}=\xi$ for some $t \geq 0$ then $c(\xi, \eta)$ denotes the instantaneous rate at which $\xi_{t}$ may change to state $\eta$. The generator of this process is

$$
\Omega f(\xi)=\sum_{\eta} c(\xi, \eta)(f(\eta)-f(\xi))
$$

where $f$ is a continuous function on $S$. Thus,

$$
\frac{d}{d t} E f\left(\xi_{t}\right)=E \sum_{\eta} c(\xi, \eta)\left(f(\eta)-f\left(\xi_{t}\right)\right)
$$

We further assume the existence of an underlying mixing/pair-formation matrix $\left(p_{i j}(\xi), q_{j i}(\xi)\right)=\left(p_{i j}^{m f}(\xi), q_{j i}^{f m}(\xi)\right)$ of the type described by Lubkin and CastilloChavez in this series. To specify the flip rates, we use the following notation. For $\xi \in S, A \subset X, B \subset X$, and $A \cap B=\emptyset$, we define $\xi_{B}^{A} \in S$ as

$$
\xi= \begin{cases}\xi(x)+1 & \text { if } x \in A \\ \xi(x)+1 & \text { if } x \in A \\ \xi(x) & \text { otherwise }\end{cases}
$$

If we change the notation slightly and let $m$ and $f$ denote the parameters associated with uninfected males and females, and $M$ and $F$ denote those associated with infected males and females, then we can define the flip rate $c(.,$.$) as follows$ (here $\gamma, \delta$, and $\sigma$ are constant parameters):

a) Pair formation

For $i>0, j>0$,

$$
c\left(\xi, \xi_{(i, \mu ; 0),(0 ; j, \nu)}^{(i, \mu ; j,)}\right)=b_{j}^{f}(\xi) \xi(0 ; j, \nu) p_{j i}^{f}(\xi) \frac{\xi(i, \mu ; 0)}{\xi(i, \mu ; 0)+\xi(i, 1-\mu ; 0)} ;
$$

b) Pair dissolution ( $\sigma$ denotes the pair dissolution rate)

For $i>0, j>0$,

$$
c\left(\xi, \xi_{(i, \mu ; j, \nu)}^{(i, \mu ; 0),(0 ; j, \nu)}\right)=\sigma_{\nu \mu} \xi(i, \mu ; j, \nu)
$$

c) Transmission ( $\delta$ denotes the transmission coefficient)

For $i>0, j>0$,

$$
c\left(\xi, \xi_{(i, 0 ; j, 1)}^{(i, 1 ; j, 1)}\right)=\delta_{F} \xi(i, 0 ; j, 1), \quad c\left(\xi, \xi_{(i, 1 ; j, 0)}^{(i, 1 ; j, 1)}\right)=\delta_{M} \xi(i, 1 ; j, 0) ;
$$

d) Recovery ( $\gamma$ denotes the recovery rate) 
For $i>0, j>0$,

$$
\begin{aligned}
& c\left(\xi, \xi_{(i, 0 ; j, 1)}^{(i, 0 ; j, 0)}=\gamma_{F} \xi(i, 0 ; j, 1), \quad c\left(\xi, \xi_{(i, 1 ; j, 0)}^{(i, 0 ; j, 0)}=\gamma_{M} \xi(i, 1 ; j, 0)\right.\right. \\
& c\left(\xi, \xi_{(i, 1 ; j, 1)}^{(i, 1 ; j, 0)}=\gamma_{F} \xi(i, 1 ; j, 1), \quad c\left(\xi, \xi_{(i, 1 ; j, 1)}^{(i, 0 ; j, 1)}=\gamma_{M} \xi(i, 1 ; j, 1)\right.\right.
\end{aligned}
$$

while for single infected individuals we have

$$
c\left(\xi, \xi_{(i, 1 ; 0)}^{(i, 0 ; 0)}\right)=\gamma_{M} \xi(i, 1 ; 0), \quad c\left(\xi, \xi_{(0 ; j, 1)}^{(0 ; j, 0)}\right)=\gamma_{F} \xi(0 ; j, 1) ;
$$

furthermore, for $i>0, j>0$, we have the combined recovery rate

$$
c\left(\xi, \xi_{(i, 1 ; j, 1)}^{(i, 0 ; j, 0)}\right)=\gamma_{F M} \xi(i, 1 ; j, 1) ;
$$

e) Removal ( $\mu$ denotes the removal rate from sexual activity)

For $i>0, j>0, \mu, \nu$,

$$
c\left(\xi, \xi_{(i, \mu ; j, \nu)}^{(i, \mu ; 0)}\right)=\mu_{f} \xi(i, \mu ; j, \nu), \quad c\left(\xi, \xi_{(i, \mu ; j, \nu)}^{(0 ; j ; \nu)}\right)=\mu_{m} \xi(i, \mu ; j, \nu) ;
$$

while for the removal rate of single individuals we have

$$
c\left(\xi, \xi_{(i, \mu ; 0)}\right)=\mu_{m} \xi(i, \mu ; 0), \quad c\left(\xi, \xi_{(0 ; j, \nu)}\right)=\mu_{f} \xi(0 ; j, \nu)
$$

f) Recruitment (ail recruited individuals are susceptible)

For $i>0, j>0$,

\section{g) Other}

$$
c\left(\xi, \xi^{(i, 0 ; 0)}\right)=\Lambda_{i}^{m}, \quad c\left(\xi, \xi^{(0 ; j, 0)}\right)=\Lambda_{j}^{f}
$$

For any other $\eta \neq \xi$, we assume $c(\xi, \eta)=0$, and $c(\xi, \xi)=-\sum_{\xi \neq \eta} c(\xi, \eta)$.

As the time $t$ changes, singles may form pairs, pairs may dissolve, and the disease may be transmitted (usually only within clearly specified pairings). The system $\left\{\xi_{t}\right\}$ consists of a series of changing elements in the set $X$, the set of all functions on $S$. The dynamics of the system is described by the rates at which the system changes. These rates are given as a set of nonnegative numbers $\{c(\xi, \eta): \xi \neq \eta, \xi, \quad \eta \in X\}$. Specifically, each $c(\xi, \eta)$ is the rate at which the system changes from $\xi$ to $\eta$, that is,

$$
P\left(\xi_{t+h}=\eta \mid \xi_{t}=\xi\right)=c(\xi, \eta) h+0(h), \forall t \geq 0 .
$$

The deterministic model that corresponds to the above stochastic model in the context of a STD such as gonorrhea (susceptible $\rightarrow$ infected $\rightarrow$ susceptible) but that incorporates couples (transmission can only occur in a couple where one of the partners is infected) is described below (for details see [4]).

Consider a population of sexually active heterosexual individuals divided into subpopulations by such factors as sex, race, socio-economic background, and average degree of sexual activity. There are $N^{f}$ female and $N^{m}$ male subpopulations, each divided into two epidemiological classes for single individuals: $f_{j}(t)$ and $m_{i}(t)$ (single susceptible females and males, at time $t$ ), and $F_{j}(t)$ and $M_{i}(t)$ (single in- 
fected females and males), all for $j=1, \ldots, N^{f}$ and $i=1, \ldots, N^{m}$. Hence the sexually-active single individuals of each sex and each subpopulation are given by $T_{j}^{f}=f_{j}+F_{j}$ and $T_{i}^{m}=m_{i}+M_{i}$. The epidemiological classes for pairs are given by $\pi_{j i}^{f m}, \pi_{j i}^{F m}, \pi_{j i}^{f M}, \pi_{j i}^{F M}$, which are, respectively, the numbers of pairs of $f$-with$m, F$-with- $m, f$-with- $M$, and $F$-with- $M$ individuals. Transmission can only occur among those individuals in pair types $\pi_{j i}^{F m}$ or $\pi_{j i}^{f M}$. Since $\pi_{j i}^{f M}=\pi_{i j}^{M f}$, we need only consider four types of pairs. We assume that the transmission probability per unit time is constant within each pair containing one infected individual. We let $\delta_{M}$ and $\delta_{F}$ be the rates for male-to-female and female-to-male transmission, respectively. The per capita recovery rates are $\gamma_{M}$ and $\gamma_{F}$ for infected males and infected females, respectively, when their partner is uninfected. When both partners are infected ( $F$-with- $M$ pairs), simultaneous treatment of both is the norm for gonorrhea, so we incorporate "combined" recovery rate $\gamma_{F M}$, with both parties moving directly to the $f$-with- $m$ (no infection) pair type. The per capita dissolution rates are $\sigma_{f m}, \sigma_{f M}, \sigma_{F m}$, and $\sigma_{F M}$ for the different types of pairs, and the per capita removal rates from sexual activity due to death or other causes are $\mu_{f}$ and $\mu_{m}$ for all females and all males, respectively. Let $\Lambda_{j}^{f}$ and $\Lambda_{i}^{m}$ denote the recruitment rates (assumed constant) of single (assumed uninfected) individuals in the female and male populations, respectively. Let $c_{j}^{f}$ and $c_{i}^{m}$ denote the rates of pair formation for females of type $j$ and males of type $i$, respectively. We use the notation

$p_{j i}^{x m} \equiv \frac{m_{i}}{M_{i}+m_{i}} p_{j i}^{f}, \quad p_{j i}^{x M} \equiv \frac{M_{i}}{M_{i}+m_{i}} p_{j i}^{f},-\cdot p_{i j}^{y f} \equiv \frac{f_{i}}{F_{i}+f_{i}} p_{i j}^{m}, \quad p_{i j}^{y F} \equiv \frac{F_{i}}{F_{i}+f_{i}} p_{i j}^{m}$,

$\left(x=f\right.$ or $F$, and $y=m$ or $M$, for $i=1, \ldots, N^{m}$ and $\left.j=1, \ldots, N^{f}\right)$ for the fraction of pair formations between the specified subgroups ( $i$ and $j$ ) wich are of given infection status; for example, $p_{j i}^{f m}$ and $p_{j i}^{f M}$ give the fractions involving uninfected $\left(m_{i}\right)$ and infected $\left(M_{i}\right)$ males, respectively. Then the gonorrhea pair formation/dissolution model is

$$
\begin{aligned}
& \frac{d f_{j}}{d t}=\Lambda_{j}^{f}+\gamma_{F} F_{j}+\left[\mu_{M}+\sigma_{f M}\right] \sum_{i=1}^{N^{m}} \pi_{j i}^{f M}+\left[\mu_{m}+\sigma_{f m}\right] \sum_{i=1}^{N^{m}} \pi_{j i}^{f m}-\left[c_{j}^{f}+\mu_{f}\right] f_{j}, \\
& \frac{d m_{i}}{d t}=\Lambda_{i}^{m}+\gamma_{M} M_{i}+\left[\mu_{F}+\sigma_{F m}\right] \sum_{j=1}^{N^{f}} \pi_{j i}^{F m}+\left[\mu_{f}+\sigma_{f m}\right] \sum_{j=1}^{N^{f}} \pi_{j i}^{f m}-\left[c_{i}^{m}+\mu_{m}\right] m_{i}, \\
& \frac{d F_{j}}{d t}=\left[\mu_{m}+\sigma_{F m}\right] \sum_{i=1}^{N^{m}} \pi_{j i}^{F m}+\left[\mu_{m}+\sigma_{F M}\right] \sum_{i=1}^{N^{m}} \pi_{j i}^{F M}-\left[c_{j}^{f}+\gamma_{F}+\mu_{f}\right] F_{j}, \\
& \frac{d M_{j}}{d t}=\left[\mu_{f}+\sigma_{f M}\right] \sum_{j=1}^{N^{f}} \pi_{j i}^{f M}+\left[\mu_{f}+\sigma_{F M}\right] \sum_{j=1}^{N^{f}} \pi_{j i}^{F M}-\left[c_{i}^{m}+\gamma_{M}+\mu_{M}\right] M_{i},
\end{aligned}
$$




$$
\begin{aligned}
& \frac{d \pi_{j i}^{f m}}{d t}=c_{j}^{f} p_{j i}^{f m} f_{j}+\gamma_{M} \pi_{j i}^{f M}+\gamma_{F} \pi_{j i}^{F m}+\gamma_{F} M \pi_{j i}^{F M}-\left[\mu_{f}+\mu_{m}+\sigma_{f m}\right] \pi_{j i}^{f m}, \\
& \frac{d \pi_{j i}^{F m}}{d t}=c_{j}^{f} p_{j i}^{F m} F_{j}+\gamma_{M} \pi_{j i}^{F M}-\left[\mu_{f}+\mu_{m}+\sigma_{F m}+\delta_{F}+\gamma_{F}\right] \pi_{j i}^{F m}, \\
& \frac{d \pi_{j i}^{f M}}{d t}=c_{j}^{f} p_{j i}^{f M} f_{j}+\gamma_{F} \pi_{j i}^{F M}-\left[\mu_{f}+\mu_{m}+\sigma_{f M}+\delta_{M}+\gamma_{M}\right] \pi_{j i}^{f M}, \\
& \frac{d \pi_{j i}^{F M}}{d t}=c_{j}^{f} p_{j i}^{F M} F_{j}+\delta_{F} \pi_{j i}^{F m}+\delta_{M} \pi_{j i}^{f M}-\left[\mu_{f}+\mu_{m}+\sigma_{F M}+\gamma_{M}+\gamma_{F}+\gamma_{F M}\right] \pi_{j i}^{F M},
\end{aligned}
$$

with initial conditions $f_{j}(0)>0, \quad m_{i}(0)>0, \quad \pi_{j i}^{f m}(0)=0, \quad \pi_{j i}^{f M}(0)=0$, $\pi_{j i}^{F m}(0)=0, \quad \pi_{j i}^{F M}(0)=0$, and at least one of the $F_{j}(0)$ and $M_{i}(0)$ greater than zero (for $i=1, \ldots, N^{m}$ and $j=1, \ldots,{ }_{N}^{f}$ ).

We invoke the results of Kurtz $[19,20]$ and conclude that for large populations the deterministic and the expected value of the associated stochastic process will have the same asymptotic behavior.

In the next section we outline the simulation process of a general stochastic process such as the one outlined in this section. In addition, for illustration purposes, we provide the results of several simulations of a particular case - the case when there is no infection.

\section{Simulation of the process $\left\{\xi_{t}: t \geq 0\right\}$}

The general approach for simulating jump Markov processes is as follows. From the construction of the flip rates we know that

$$
c(\xi)=\sum_{\eta \in S} c(\xi, \eta)<\infty .
$$

Hence, if we let the sequence $0=\rho_{0}<\rho_{1}<\rho_{2}<\ldots$ denote the jump times of the process, then $\tau_{n}=\rho_{n}-\rho_{n-1}$ has an exponential distribution with rate $c\left(\xi_{\beta_{n-1}}\right)$. We can simulate the process using the following procedure:

1. First, set the initial state $\xi_{0}$ and assume that a sequence of $n$ jump times $0=\rho_{0}<\rho_{1}<\ldots<\rho_{n}$ and their corresponding states $\xi_{\beta_{i}}, 1 \leq i \leq n$ have been determined.

2. Get $\tau_{n+1}$ from $\exp \left\{\xi_{\beta_{n}}\right\}$ and let $\beta_{n+1}=\beta_{n}+\tau_{n}$.

3. Set $\xi_{\beta_{n+1}}=\eta$ with probability $c\left(\xi_{\beta_{n}, \eta}\right) / c\left(\xi_{\beta_{n}}\right)$

4. Define $\xi_{t}=\xi_{\beta_{n}}$ for $\beta_{n} \leq t<\beta_{n+1}$.

We proceed to simulate the above stochastic model in a very special situation. We assume that the infection rates $\delta_{M}$ and $\delta_{F}$ are equal to zero or, equivalently, that there are no infected individuals in the population. Hence, we are simulating a purely demographic model. Individuals form and dissolve pairs. There is constant recruitment into and removal from the ranks of the sexually active, and we 
have individuals of several types (e.g., economic or social classes). The simulation, described below, will have as its average dynamics the corresponding deterministic dynamics. However, the use of a stochastic framework allows us to study the changes in variance as a function of time.

Simulations were carried out using four groups of single males $\left(m_{1}, m_{2}, m_{3}, m_{4}\right)$ and four groups of single females $\left(f_{1}, f_{2}, f_{3}, f_{4}\right)$, resulting in 16 possible pairing types $\left(m_{1} f_{1}, m_{1} f_{2}, \ldots, m_{2} f_{1}, m_{2} f_{2}, \ldots, m_{4} f_{4}\right)$. The initial numbers of single males and females, by group, as well as such groups' recruitment, pair formation, and removal rates are shown in Table 1.

Table 1. Single males and single females: Number of individuals and parameters used in stochastic simulations

\begin{tabular}{ccccc}
\hline Group & $\begin{array}{c}\text { Group } \\
\text { Size }\end{array}$ & $\begin{array}{c}\text { Recruitment } \\
\text { Rate }\end{array}$ & $\begin{array}{c}\text { Pair Formation } \\
\text { Rate }\end{array}$ & $\begin{array}{c}\text { Removal } \\
\text { Rate }\end{array}$ \\
\hline$m_{1}$ & 1000 & 100 & 3.500 & 0.1 \\
$m_{2}$ & 900 & 90 & 3.000 & 0.1 \\
$m_{3}$ & 800 & 80 & 2.500 & 0.1 \\
$m_{4}$ & 2700 & 270 & 3.500 & 0.1 \\
$f_{1}$ & 2000 & 200 & 2.500 & 0.1 \\
$f_{2}$ & 1000 & 100 & 2.450 & 0.1 \\
$f_{3}$ & 500 & 50 & 2.300 & 0.1 \\
$f_{4}$ & 3500 & 350 & 2.586 & 0.1 \\
\hline
\end{tabular}

The initial number of pairs was constrained to zero for all possible pairing types. Removal rates for paired individuals-just as those for singles-were held constant at 0.1. For this investigation, the pair dissolution rate, $\sigma$, was assumed as invariant within the scope of any one simulation (with any number of realizations), and different pair dissolution rates were introduced for different sets of simulations. We started by using a value of $\sigma=5$, and then tested, first, larger values $(5<\sigma \leq 20)$ and then smaller ones $(0 \leq \sigma<5)$.

A set of runs with 10,000 realizations each was allowed to simulate the process up to time $t=2$. Another set of runs, but with only 1,000 realizations each, was extended to $t=32$ in order to test the consistency of those trends observed in the shorter runs. ${ }^{1}$

As shown in Figure 1, stability in all of the groups' absolute sizes is reached early-at $t \approx 0.5$ when $\sigma=5$ (and even earlier when $\sigma>5$; relative distributions, not shown, corroborate the same pattern). This trait of very rapid stabilization is maintained when average group sizes are computed from simulations based on as few as 30 realizations. As $\sigma \rightarrow 0$, the time required to attain stability increases, but only marginally. Even in the extreme case where $\sigma=0$, i.e., when pair dissolution

1 All simulations were conducted on Quadra 950 and Quadra 700 Macintosh computers. We used a custom-developed Pascal program optimized for the specific design analyzed here (with four single male groups, four single female groups, and 16 pairing types). Typical simulations took between two and thirty minutes to complete. The lengthiest ones ran for several hours. 
is not allowed in the model, stability is reached at $t \approx 1.5$, if not sooner (see Figure 2).

Variance trends expose a different and somewhat mixed story. Standard deviations for all pairing types tend to settle down at roughly the same time as do mean values. For instance, stability is reached at $t \approx 0.5$ when $\sigma=5$ (see Figure 3). As shown in Figure 4, however, variation for each of the groups of single individuals, male and female, continues to increase at least until $12 \leq t \leq 16$. Even 1,000 realizations were not enough to provide a clear cut picture of variance patterns after such time.

Further work should allow us to ascertain whether the patterns observed beyond $t=12$ in Figure 4 indeed reflect stability-or only smaller increments in variation. Simulations with 10,000 or more realizations and a time horizon of $50 \leq t \leq 100$ will be necessary to accomplish this. We also plan to callibrate, in addition to $\sigma$, the values of pair formation and recruitment parameters. We may thus assess the effects such rates have on the magnitude of variation and on the onset of stability.

\section{Conclusions}

In this article we have introduced a flexible stochastic framework for the modeling of contact structures in biology. We have provided modeling applications in demography (including demographic models for pairs) and in epidemiology. It is clear that applications of stocahstic models to the inheritance of cultural traits and to food web dynamics - such as the processes described using deterministic models in this series - are also possible.

This research represents our initial efforts in understanding the role of social structures in disease dynamics where stochasticity plays a major role. Most deterministic models in the past assumed a fixed social/behavioral structure. Most stochastic models used the mass-action law. The study of the transmission dynamics of HIV highlighted the limitations of some of these approaches. We have observed a large number of theoretical advances over the last few years (see CastilloChavez, [7]; Jewell et al. [16]; Anderson and May [2]; and Hethcote and Van Ark [15]). However, we have just begun to understand the effects of changing contact structures in population dynamics.

\section{Acknowledgements}

This research was partially supported by funds from the Dean and the Office of Sponsored Programs of the College of Agriculture and the Mathematics Science Institute at Cornell University, and by NSF grant DEB-9253570 to Carlos CastilloChavez. This work was completed while Carlos Castillo-Chavez was a visiting member of the Isaac Newton Institute, Cambridge University. 


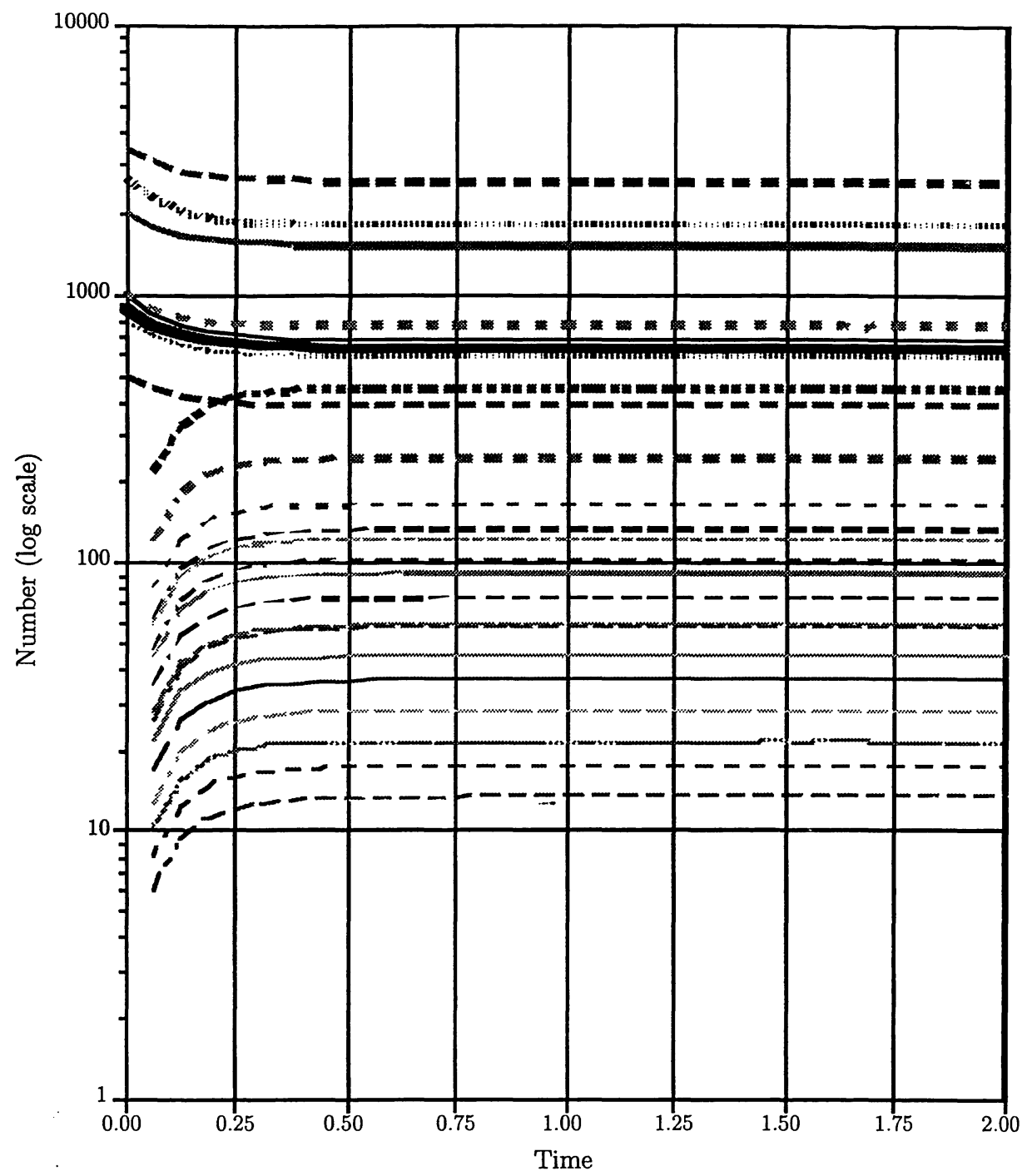

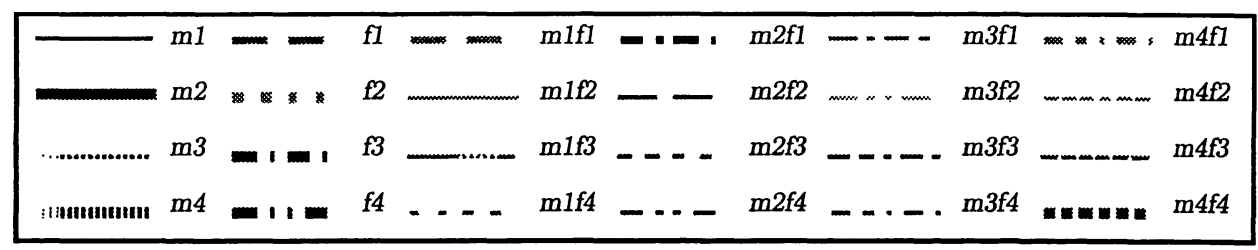

Figure 1. Mean population by sex, class, and pair type. Simulation with $\sigma=5, t \leq 2$, and 10,000 realizations 


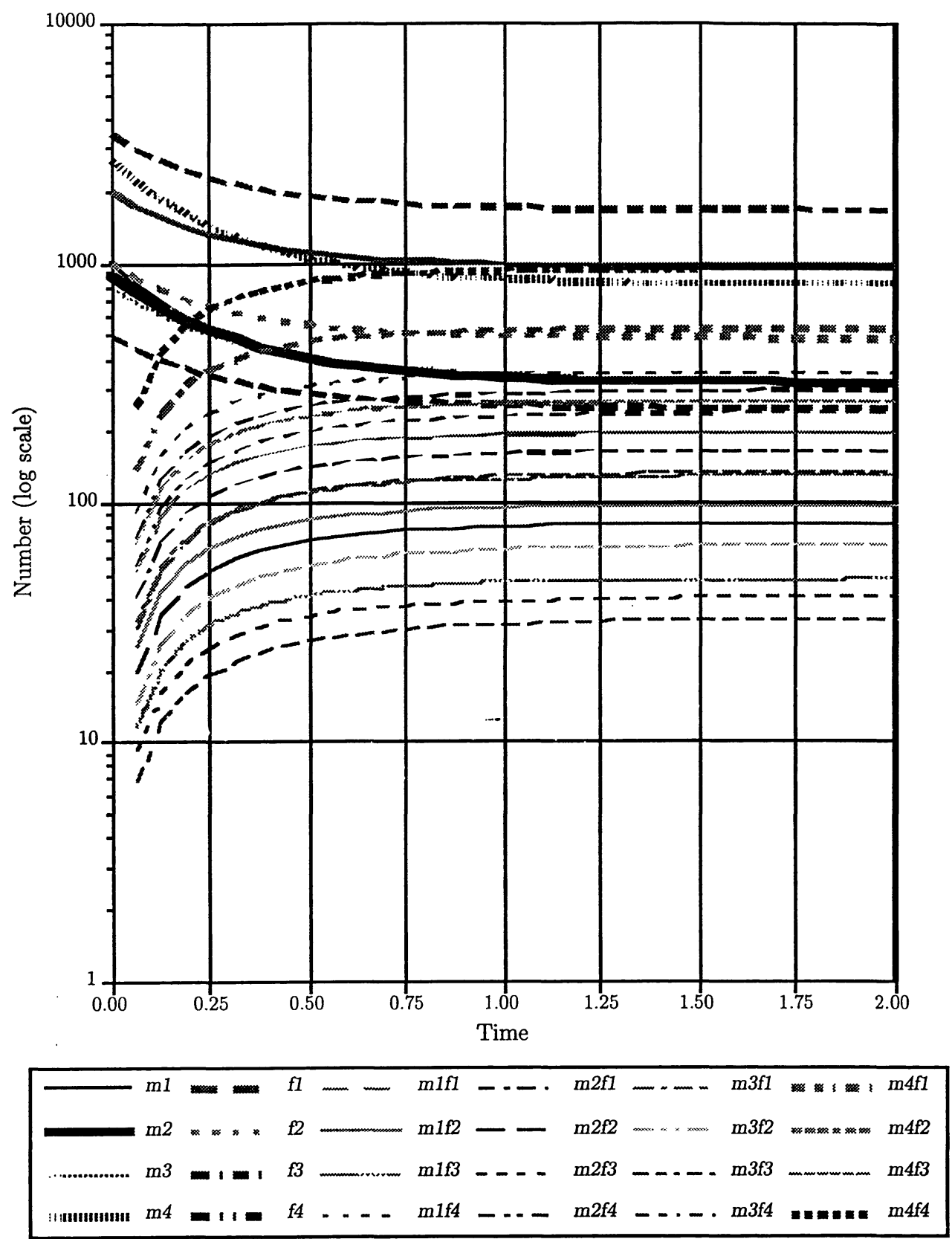

Figure 2. Mean population by sex, class, and pair type. Simulation with $\sigma=0, t \leq 2$, and 10,000 realizations 


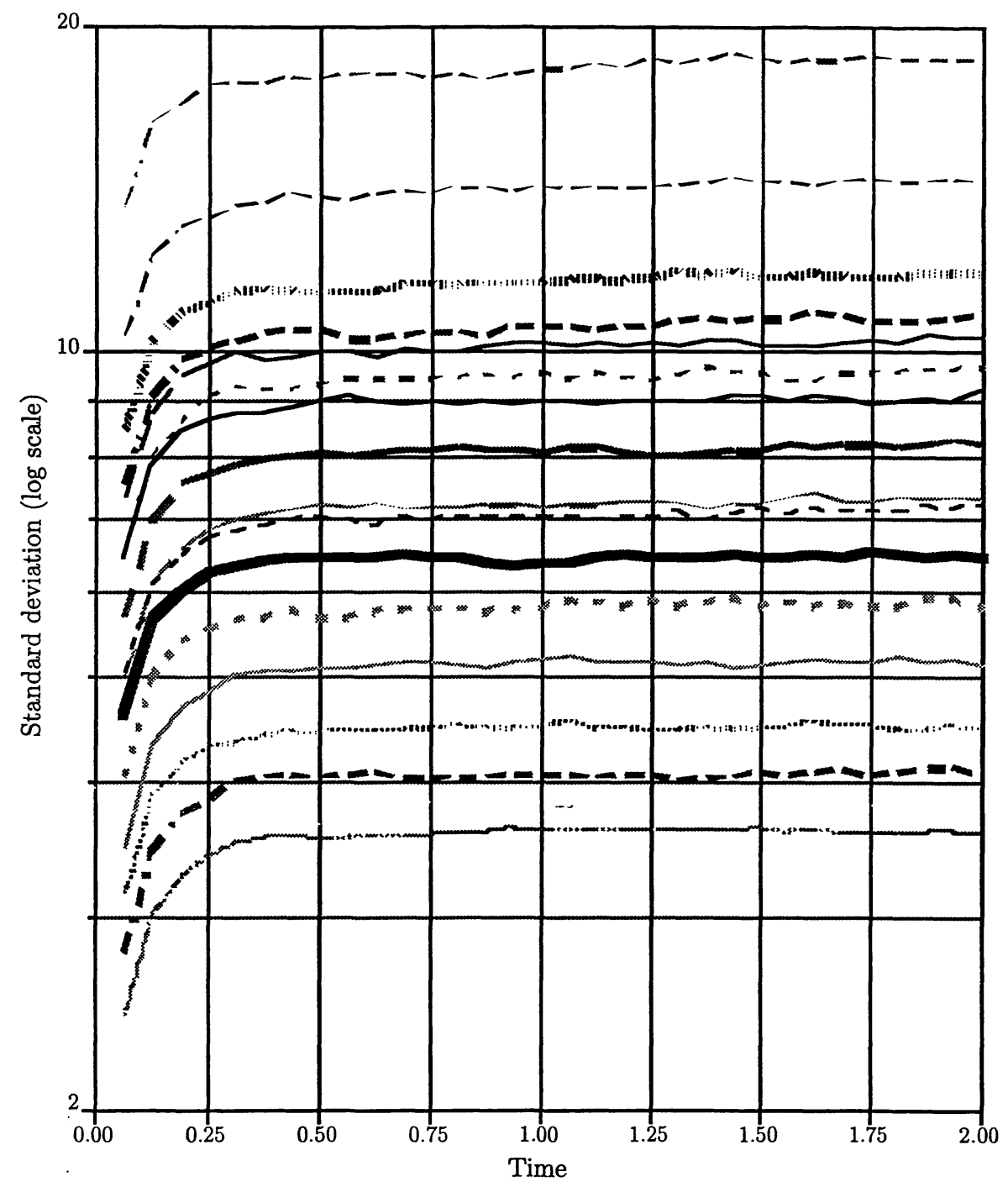

\begin{tabular}{|c|c|c|c|c|c|c|c|}
\hline & $m 1 f 1$ & m.m & $m 2 f 1$ & س & $m 3 f 1$ & $-\cdots$ & $m 4 f 1$ \\
\hline & m1f2 & $\approx=$ & $m 2 f 2$ & nomm & m3f2 & -- & $m 4 f 2$ \\
\hline ............e & m1f3 & $=10$ & $m 2 f 3$ & $\cdots$ & m3f3 & - & $m 4 f 3$ \\
\hline u::1::an & m1f4 & $=11$ & $m 2 f 4$ & - - & $m 3 f 4$ & - & $m 4 f 4$ \\
\hline
\end{tabular}

Figure 3. Standard deviations over time by pair type. Simulation with $\sigma=5$, $t \leq 2$, and 10,000 realizations 


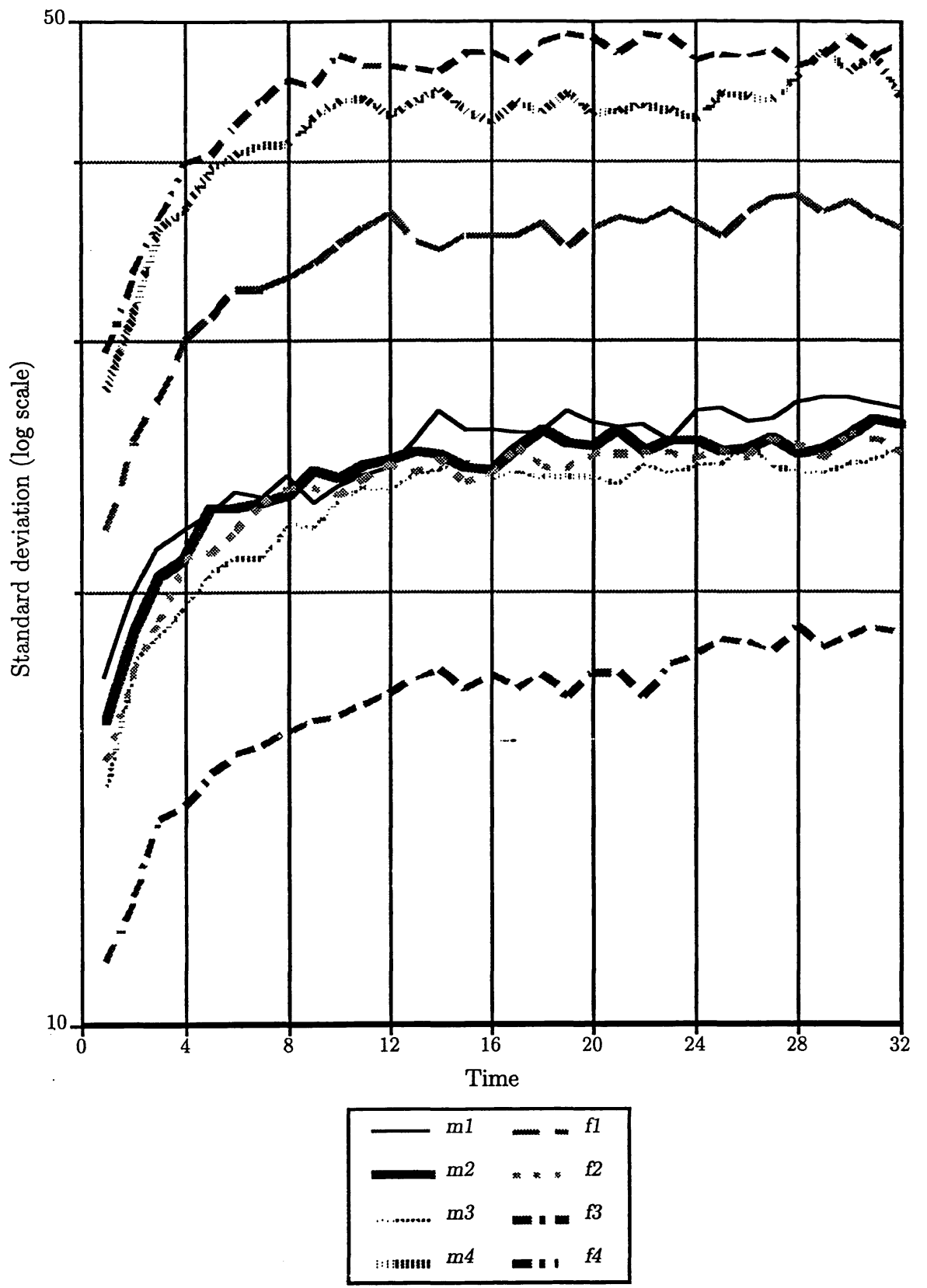

Figure 4. Standard deviations over time by sex and class. Simulation with $\sigma=5, t \leq 32$, and 1,000 realizations 


\section{References}

[1] Anderson, R.M., ed. Population Dynamics of Infectious Diseases: Theory and Applications. Chapman and Hall, London-New York 1982.

[2] Anderson, R.M., and R.M. May. Infectious Diseases of Humans. Oxford Science Publications, Great Britain 1991.

[3] Bailey, N.T.J. The Mathematical Theory of Infectious Diseases and Its Applications. Griffin, London 1975.

[4] Blythe, S.P., C. Castillo-Chavez, J. Palmer, and M. Cheng. Towards a unified theory of mixing and pair formation. Math. Biosci. 107 (1991), 379-405.

[5] Busenberg, S., and C. Castillo-Chavez. Interaction, Pair Formation and Force of Infection Terms in Sexually Transmitted Diseases (Lect. Notes Biomath. 83). Springer-Verlag, New York 1989.

[6] Busenberg, S., and C. Castillo-Chavez. A general solution of the problem of mixing subpopulations, and its application to risk- and age-structured epidemic models for the spread of AIDS. IMA J. of Mathematics Applied in Med. and Biol. 8 (1991), 1-29.

[7] Castillo-Chavez, C., ed. Mathematical and Statistical Approaches to AIDS Epidemiology (Lect. Notes Biomath. 83). Springer-Verlag, New York 1989.

[8] Dietz, K. On the transmission dynamics of HIV. Math. Biosci. 90 (1988), 397-414.

[9] Dietz, K., and K.P. Hadeler. Epidemiological models for sexually transmitted diseases. J. Math. Biol. 26 (1988), 1-25.

[10] Fredrickson, A.G. A mathematical theory of age structure in sexual populations: Random mating and monogamous marriage models. Math. Biosci. 20 (1971), 117143.

[11] Hadeler, K.P. Pair formation in age-structured populations. Acta Applicandae Mathematicae 14 (1989a), 91-102.

[12] Hadeler, K.P. Modeling AIDS in structured populations. 47th Session of the International Statistical Institute, Paris, August/September. Conf. Proc., C1-2 (1989b), 83-99.

[13] Hadeler, K.P., and K. Nagoma. Homogeneous models for sexually transmitted diseases. Rocky Mountain Journal of Mathematics. 20 (1990), 967-986.

[14] Hethcote, H.W., and J.A. Yorke. Gonorrhea Transmission Dynamics and Control (Lect. Notes Biomath. 56). Springer-Verlag, New York 1984.

[15] Hethcote, H.W., and J.W. Van Ark. Modeling HIV Transmission and AIDS in the United States (Lect. Notes Biomath. 95). Springer-Verlag, New York 1992.

[16] Jewell, N.P., K. Dietz, and V.T. Farewell. AIDS Epidemiology: Methodological Issues. Birkhäuser, Boston-Basel-Berlin 1991.

[17] Kendall, D.G. Stochastic processes and population growth. Roy. Statist. Soc., Ser. B2 (1949), 230-264.

[18] Keyfitz, N. The mathematics of sex and marriage. Proceedings of the Sixth Berkeley Symposium on Mathematical Statistics and Probability. 4 (1949), 89-108.

[19] Kurtz, T.G. Solutions of ordinary differential equations as limits of pure jump Markov processes. J. Appl. Probab. 7 (1970), 49-58.

[20] Kurtz, T.G. Limit theorems for sequences of jump Markov processes approximating differential equations. J. Appl. Probab. 8 (1971), 344-56.

[21] Leslie, P.H. On the use of matrices in certain population mathematics. Biometrika, 33 (1945), 183-212. 
[22] Lotka, A.J. The stability of the normal age distribution. Proceedings, National Academy of Sciences, 8 (1922), 339-345.

[23] Luo, X., and C. Castillo-Chavez. Limit behavior of pair formation models with large dissolution rates. Journal of Mathematical Systems, Estimation, and Control (1993, in press).

[24] Luo, X., C. Castillo-Chavez, and M. Cheng. A probabilistic model of pair formation (Biometrics Unit Technical Report, BU-1145-M, 1991).

[25] MacKendrick, A.G. Applications of mathematics to medical problems. Proceedings Edinburgh Math. Soc. 44 (1926), 98-130.

[26] McFarland, D.D. Comparison of Alternative Marriage Models. In: Population Dynamics (ed. by T.N.E. Greville), 89-106. Academic Press, New York-London 1972.

[27] Parlett, B. Can There Be a Marriage Function? In: Population Dynamics (ed. by T.N.E. Greville), 107-135. Academic Press, New York-London 1972.

[28] Pollard, J.H. Mathematical Models for the Growth of Human Populations. Cambridge University Press, Cambridge 1973.

[29] Ross, R. The Prevention of Malaria (2nd edition, with addendum). John Murray, London 1911.

[30] Waldstätter, R. Pair Formation in Sexually Transmitted Diseases. (Lect. Notes Biomath. 83, 260-274). Springer-Verlag, New York 1989. 